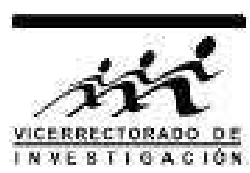

\title{
Un modelo de elementos finitos de la hidrodinámica alrededor de hidrofolios
}

\author{
C. A. A. Carbonel* \\ Facultad de Ciencias Físicas, Universidad Nacional Mayor de San Marcos, Lima, Perú
}

Recibido 7 octubre 2015 - Aceptado 29 octubre 2015

\begin{abstract}
En este artículo se presenta un modelo de elementos finitos para predecir el comportamiento del agua en movimiento alrededor de hidrofolios. El modelo propuesto resuelve las ecuaciones de conservación de momentum y masa incluyendo términos de advección, presión y esfuerzo cortante. Para la descripción de la influencia de la turbulencia, el modelo de Prandtl-Kolgomorov es considerado. Una ecuación de transporte es incluida en la formulación para simular la evolución de la fracción de vapor de agua. Condiciones de frontera débilmente reflectantes son impuestas en las fronteras abiertas de entrada y salida del flujo para evitar las intensas reflecciones de las ondas de presión que generan inestabilidades. Diversos experimentos numéricos son realizados usando el perfil hidrodinámico NACA 0012. Los resultados no permanentes del modelo reproducen las características esenciales de los flujos alrededor de los hidrofolios en diversos casos. El modelo es capaz de describir la mezcla de líquido y vapor cuando la presión esta por debajo de la presión de vapor en el caso de cavitación.
\end{abstract}

Palabras claves: Elementos finitos, hidrodinámica, flujos de mezcla, cavitación, hidrofolios.

\section{A finite element model of the Hydrodynamics around hydrofoils}

In this paper, a finite element model is presented to predict the hydrodynamics around hydrofoils. The proposed model solves the equations of conservation of mass and momentum, including advection, pressure and shear stress terms. To describe the influence of turbulence, the PrandtlKolmogorov model is considered. A transport equation is included in the formulation to simulate the evolution of water vapor fraction. Weakly reflective boundary conditions are imposed on open boundaries to avoid the intense reflections of the pressure waves that generate instability. Various numerical experiments are performed using the hydrodynamic profile NACA 0012. The non permanent model results reproduce the essential characteristics of the flows around the hidrofoils in various cases. The model is able to describe the mixture of liquid and vapor when the pressure is below the vapor pressure during cavitation phenomena.

Keywords: Finite elements, Hydrodynamics, mixture flows, cavitation, hydrofoils.

El movimiento del fluido alrededor de los cuerpos sumergidos abarcan una variedad de fenómenos en mecánica de fluidos y tiene una amplia variedad de aplicaciones en ingeniería, tales como el flujo en turbinas y en vehículos sumergidos. El escurimiento fluido alrededor de un cuerpo produce flujos complejos porque la cinemática y las fuerzas producidas dependen en gran medida de diversos parámetros tales como el tamaño, la orientación, la velocidad y las propiedades de los fluidos. La presión resultante y el campo de velocidades alrededor del cuer-

*ccarbonelh@unmsm.edu.pe 
po se modifica debido a la geometría y la fricción en las fronteras del cuerpo. Cuando el número de Reynolds aumenta, el flujo comienza a separarse con la formación de movimientos vorticosos inestables principalmente detrás del cuerpo [1]. El comportamiento turbulento del fluido es una pregunta abierta y existe diferentes opciones para modelar la turbulencia. Las ecuaciones turbulentas más acostumbradas son de la familia de modelos $k-\epsilon$. Un problema de este tipo de modelos es que son dependientes de la geometría considerada y también sufren de las deficiencias de la hipótesis del gradiente [2]. Debido a este hecho, la presencia de muchas constantes, que no son universales, es la característica de estos modelos. Esto afecta la estabilidad de las soluciones que sumadas a la problemática de las reflecciones en las fronteras abiertas requiere de la inclusión de filtros y estabilizadores sin base física para obtener soluciones estables. A pesar de esto, se utiliza con frecuencia en muchos paquetes de softwares. Otra opción es el uso de ecuaciones llamadas de cero o uno, por ejemplo, el modelo Smagorinsky o el modelo Prandtl-Kolmogorov.

En la literatura, se encuentran soluciones numéricas de los movimientos del agua sobre hidroplanos o hidrofolios generalmente utilizando las técnicas de volúmenes finitos y diferencias finitas [3-5]. El método de elementos finitos es una técnica prácticamente inexplorada para estos fines. El presente trabajo tiene como objetivo avanzar en el estudio de este tipo de problemas no permanentes usando elementos finitos, que es una técnica numérica poderosa para resolver problemas de ingeniería [6.7].

En el presente artículo se describen los avances realizados en el estudio del campo de flujo alrededor de hidrofolios para diferentes velocidad de flujo y condiciones de presión [8], incluyendo los casos con interacciones entre turbulencia, cambios de fase y variaciones de presión.

\section{Ecuaciones gobernantes}

Estudiamos la dinámica del flujo sobre un hidrofolio, en la superficie del cual, frontera $\Gamma_{H}$, se asume condiciones no deslizantes. El hidrofolio está situado dentro de un canal sumergido de longitud $L$ y ancho $0.4 L$, con fronteras abiertas, frontera $\Gamma_{E}$, solamente en el eje longitudinal, $x_{1}=0$ y $x_{1}=L$. Las otras fronteras del canal son paredes cerradas, frontera $\Gamma_{C}$.

Para la descripción matemática del problema hidrodinámico, se considera agua con densidad $\rho$ en un sistema coordenado vertical $\left(x_{1}, x_{2}\right)$, con componentes de velocidad $\bar{u}=\left(u_{1}, u_{2}\right)$, presión $p$, gravedad $\bar{g}=(0, g)$, viscosidad turbulenta $\nu_{T}$, derivada material $\frac{d}{d t}=\frac{\partial}{\partial t}+\bar{u} \cdot \nabla$ y operador nabla $\nabla=\left(\frac{\partial}{\partial x_{1}}, \frac{\partial}{\partial x_{2}}\right)$. Las ecuaciones de movimiento y continuidad hidrodinámicas son

$$
\begin{gathered}
\rho \frac{d \bar{u}}{d t}+\nabla p+\rho \nu_{T} \nabla \nabla \cdot \bar{u}-\rho \bar{g}=0, \\
\nabla \cdot \bar{u}=0
\end{gathered}
$$

con condiciones no deslizantes en la superficie del hidrofolio $\Gamma_{H}$,

$$
u=0 \quad \text { en } \quad \Gamma_{H} ;
$$

condiciones esenciales de frontera en la entrada del dominio $\Gamma_{E}$,

$$
u=u_{\infty} \quad \text { en } \quad \Gamma_{E} ;
$$

condiciones de frontera naturales en las paredes rígidas del dominio $\Gamma_{C}$,

$$
\frac{\partial \bar{u}_{n}}{\partial x_{n}}=0 \quad \text { en } \quad \Gamma_{C} ;
$$

y condiciones no reflectantes en las fronteras abiertas $\Gamma_{0}$,

$$
\rho \frac{\partial \bar{u}_{n}}{\partial t}+\nabla_{n} p=0 \quad \text { en } \quad \Gamma_{0} .
$$

La viscosidad turbulenta $\nu_{T}$ es modelada mediante la formulación de Prandtl-Kolmogorov

$$
\nu_{T}=c \rho l \sqrt{k}
$$

siendo $c=0.54, k$ es la energia cinética turbulenta y $l$ es la longitud característica.

La evaluación de $k$ se realiza mediante la ecuación

$$
\frac{d k}{d t}-\beta+\epsilon=0
$$

siendo

$$
\beta=\nabla \frac{\nu_{T}}{\sigma} \nabla k+\nu_{T}\left|\nabla \bar{u}+\nabla \bar{u}^{T}\right|^{2},
$$

el término generador de energía cinética y $\varepsilon$ la disipación de energía cinética aproximada como

$$
\varepsilon=\rho c_{\varepsilon} \frac{k^{3 / 2}}{l} .
$$

Para casos, con la presencia de gases en el agua, casos de cavitación, se describe la evolución de la fracción de masa de vapor $f$ mediante una ecuación de transporte, siguiendo la propuesta de Shingal et. al. [9], dada por

$$
\frac{d \rho f}{d t}-\left(S_{e}-S_{c}\right)=0
$$

donde $\rho$ es la densidad media, $f$ es la fraccion de masa de vapor y $S_{e, c}$ representan las fuentes de generación 
de vapor y condensación de acuerdo a la ecuación de Rayleigh-Plesset [3, 4],

$$
\begin{gathered}
S_{e}=C_{e} \frac{\sqrt{k}}{\sigma} \rho_{l} \rho_{v} \sqrt{\frac{2}{3} \frac{P_{v}-P}{\rho_{l}}}(1-f), \quad P<P_{v}, \\
S_{c}=C_{c} \frac{\sqrt{k}}{\sigma} \rho_{l} \rho_{v} \sqrt{\frac{2}{3} \frac{P-P_{v}}{\rho_{l}}} f, \quad P>P_{v},
\end{gathered}
$$

en donde $P_{v}$ es la presión de vapor de saturación del agua.

La relación entre la densidad de mezcla $\rho$ y $f$ es la siguiente:

$$
\frac{1}{\rho}=\frac{f_{v}}{\rho_{v}}+\frac{1-f_{v}}{\rho_{l}} .
$$

Para fines del problema actual se define la fracción de volumen de vapor $\alpha_{v}$ relacionada a $f$ de acuerdo a

$$
\alpha_{v}=f \frac{\rho}{\rho_{v}} .
$$

\section{El modelo de elementos finitos}

La formulación del problema de contorno de la hidrodinámica no permanente consiste en encontrar $\left[\bar{u}, p, k, f_{v}\right]$ en un espacio funcional $\mathcal{S}$ tal que para un conjunto de funciones de prueba admisibles $\left[w^{u}, w^{p}, w^{k}\right.$, $\left.w^{f}\right] \in \mathcal{V}$ se satisface

$$
\begin{gathered}
\int_{\Omega} w^{u}\left\{\rho \frac{d \bar{u}}{d t}+\nabla p+\rho \nu_{T} \nabla \nabla \bar{u}-\rho \bar{g}\right\} d \Omega=0 \\
\int_{\Omega} w^{p} \nabla \bar{u} d \Omega=0 \\
\int_{\Omega} w^{k}\left\{\frac{d k}{d t}-\beta+\epsilon\right\} d \Omega=0 \\
\int_{\Omega} w^{f}\left(\frac{\partial \rho f}{\partial t}+\nabla f \rho \bar{u}-S_{e}+S_{c}\right) d \Omega=0
\end{gathered}
$$

y condiciones complementarias en $\Gamma_{0}$,

$$
\int_{\Gamma_{0}} w^{u}\left[\rho \frac{\partial \bar{u}_{n}}{\partial t}+\nabla_{n} p\right]_{t^{n}}^{t^{n+1}} d \Gamma=0 \quad \text { en } \quad \Gamma_{0}
$$

siendo $\bar{u}_{n}$ la componente normal de velocidad en la frontera abierta.

La discretizacion consiste en introducir las expansiones de los elementos finitos

$$
\begin{gathered}
u_{1}=u_{1 j}(t) \phi_{j}, u_{2}=u_{2 j}(t) \phi_{j}, p=p_{j}(t) \phi_{j}, \\
k=k_{j}(t) \phi_{j}, f=f_{j}(t) \phi_{j} ; j=1,2, \ldots, N_{\text {nod }},
\end{gathered}
$$

siendo $\bar{u}_{j}(t)=\left[u_{1 j}(t), u_{2 j}(t)\right]$ y $\phi_{j}\left(x_{1}, x_{2}\right)$ las funciones base.

Substituyendo estas aproximaciones en las variables, las ecuaciones gobernantes se escriben como

$$
\begin{gathered}
M^{u} \frac{d \bar{u}_{j}(t)}{d t}+H^{u} p_{j}(t)-R^{u} \bar{u}_{j}(t)-G_{u}=0 \\
H^{c} \bar{u}_{j}(t)=0 \\
M^{k} \frac{d k_{j}(t)}{d t}-R^{k} k_{j}(t)-P^{k}+D^{k}=0 \\
M^{f} \frac{d f_{j}(t)}{d t}-S^{f}=0 \\
\text { para } \quad j=1,2, \ldots, N_{\text {nod }} .
\end{gathered}
$$

Las matrices $M^{u}, M^{k}, M^{f}, H^{u}, H^{c}, R^{u}, R^{k}$ y los vectores $G_{u}, P^{k}, D^{k}, S^{f}$ representan los términos compuestos por las integrales consideradas en la formulación variacional,

$$
\begin{array}{r}
M^{u}=\int_{\Omega} w^{u} \rho\left(\phi_{j}, \phi_{j}\right) d \Omega \\
H^{u}=\int_{\Omega} w^{u} \nabla\left(\phi_{j}, \phi_{j}\right) d \Omega \\
R^{u}=\int_{\Omega} w^{u} \rho \nu_{T}^{u} \nabla\left(\phi_{j}, \phi_{j}\right) d \Omega \\
G^{u}=\int_{\Omega} w^{u} \rho \bar{g} d \Omega \\
H^{c}=\int_{\Omega} w^{p} \nabla\left(\phi_{j}, \phi_{j}\right) d \Omega \\
M^{k}=\int_{\Omega} w^{k} \phi_{j} d \Omega \\
R^{k}=\int_{\Omega} w^{k} \frac{1}{\sigma} \nu_{T} \nabla_{j} \nabla w^{k} \frac{1}{\sigma} \nu_{T} \nabla_{j} d \Omega \\
P^{k}=\int_{\Omega} w^{k} \nu_{T}\left|\nabla \bar{u}+\nabla \bar{u}^{T}\right|^{2} d \Omega \\
D^{k}=\int_{\Omega} w^{k} \epsilon d \Omega \\
M^{f}=\int_{\Omega} w^{f} \rho \phi_{j} d \Omega \\
S^{f}=\int_{\Omega} w^{f}\left(S_{e}-S_{c}\right) d \Omega .
\end{array}
$$

En la presente formulación, las funciones de prueba $\left[w^{u}, w^{p}, w^{k}, w^{f}\right]$ son iguales a $\phi_{j}$. La aproximación en tiempo de las derivadas es obtenida usando una descripción genérica $\mathcal{U}(t)$ con una aproximación lineal entre 2 niveles de tiempo $n$ y $n+1$ que es expresado como

$$
\mathcal{U}(t)=\theta \mathcal{U}^{n+1}+(1-\theta) \mathcal{U}^{n},
$$


donde $\theta=\frac{t-t^{n}}{t^{n+1}-t^{n}}$, y la variación temporal de $\mathcal{U}(t)$ es

$$
\frac{d}{d t} \mathcal{U}(t)=\frac{1}{\Delta t}\left(\mathcal{U}^{n+1}-\mathcal{U}^{n}\right) .
$$

En los términos de las ecuaciones resultantes, $\theta$ es fijado igual a 1.

La integración numérica tiene una estructura implícita, de esta forma tenemos que

$$
\begin{gathered}
M^{u} \frac{\left(\bar{u}_{j}^{n+1}-\widehat{u}_{j}^{n}\right)}{\Delta t}+H^{u} p_{j}^{n+1}-R^{u} \bar{u}_{j}^{n+1}-G_{u}=0 \\
H^{c} \bar{u}_{j}^{n+1}=0 \\
M^{k} \frac{\left(k_{j}^{n+1}-\hat{k}_{j}^{n}\right)}{\Delta t}-R^{k} k_{j}^{n+1}-P^{k}+D^{k}=0 \\
M^{f} \frac{\left(f_{j}^{n+1}-\hat{f}_{j}^{n}\right)}{\Delta t}-S^{f}=0
\end{gathered}
$$

en donde los términos $\widehat{u}_{j}^{n}, \widehat{p}_{j}^{n}, \widehat{k}_{j}^{n}, \widehat{f}_{j}^{n}$ son variables en el nivel $n$ obtenidas mediante el método de las características [10,11]. La implementación del sistema variacional [12] considerados en la presente subsección, usa una red de elementos finitos triangulares con fronteras abiertas y cerradas.

\section{Experimentos numéricos}

Se estudia un hidrofolio con perfil NACA 0012 y cuerda $c=0.1 \mathrm{~m}$. El hidrofolio es localizado en el medio de un tunel de agua de longitud 10c y altura 4c. La malla de elementos finitos es de alrededor de 14100 elementos y 7200 nodos (Figure 1), dependiendo del angulo de inclinación del hidrofolio. La presión referencial $p_{\infty}$ se incrementa con la profundidad. El flujo posee en la entrada, lado izquierdo, una velocidad $u_{\infty}$.

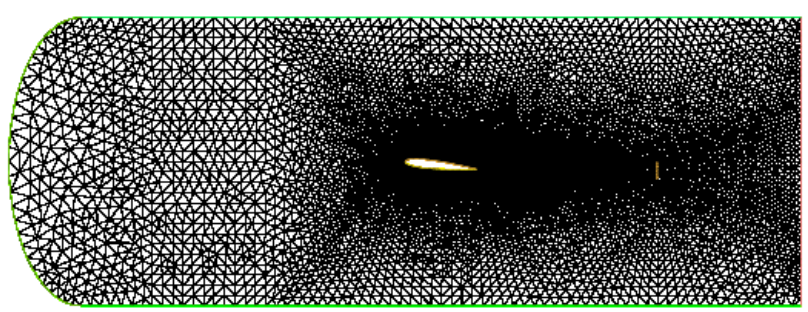

Figura 1: Malla de elementos finitos del dominio hidrodinamico con hidrofolio

En las paredes rígidas, las condiciones deslizantes son impuestas correspondiendo a las paredes superior e inferior del tunel. También en la superficie del hidrofolio se impone las condiciones no deslizantes. En las fronteras abiertas, de entrada y salida del flujo, lado izquierdo y derecho del canal, una condición debilmente reflectante es impuesta.
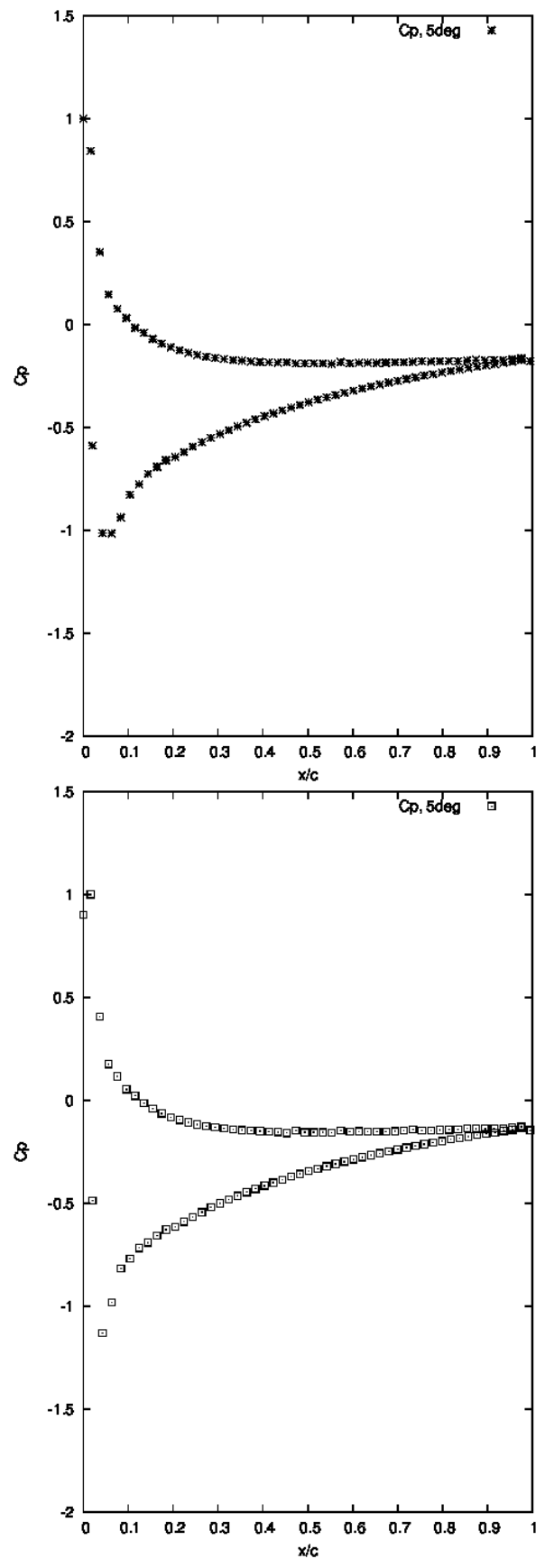

Figura 2: $C_{p}$ en la superficie del hidrofolio para $\alpha=5^{\circ}$, $u_{\infty}=1 \mathrm{~m} / \mathrm{s}$ y la profundidad, arriba 2c; abajo: $4 \mathrm{c}$.

Los parámetros usados en los experimentos son los siguientes: densidad del agua a $25^{\circ} \mathrm{C}$ es fijada como 
$\rho_{l}=997.009 \mathrm{~kg} / \mathrm{m}^{3}$, con viscosidad dinámica igual a $8.91 \times 10^{-4} \mathrm{~Pa}$-s. Para los experimentos con cavitación, la densidad del vapor del agua es $\rho_{v}=0.02308 \mathrm{~kg} / \mathrm{m}^{3}$, la presion de vaporización es $P_{v}=3169 \mathrm{~Pa}$. El intervalo de tiempo de cálculo es $\Delta t=0.5 \mathrm{~ms}$ y se integra en el tiempo hasta $500 \mathrm{~ms}$.

En los experimentos, inicialmente se estudia la circulación del agua alrededor de un hidrofolio NACA 0012 y se discute las distribuciones de presiones resultantes.

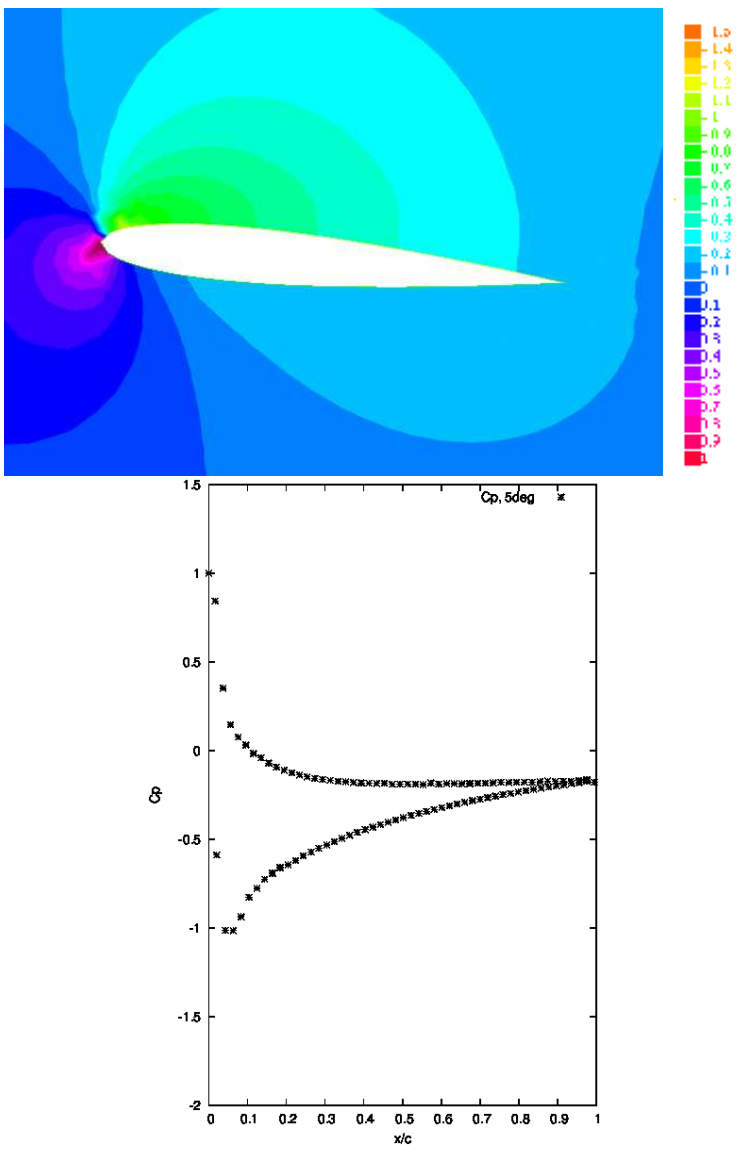

Figura 3: Campo de $C_{p}$. Arriba: para $\alpha=5^{\circ}, u_{\infty}=1 \mathrm{~m} / \mathrm{s}$, prof $=2$ c. Abajo: $C_{p}$ en la superficie del hidrofolio.

Los experimentos son realizados para diferentes profundidades de agua del hidrofolio y diferentes ángulos de ataque $\alpha$. Las profundidades de agua que hemos considerado fueron 2c y 4c. En el lado izquierdo del canal, frontera abierta, el agua ingresa con un perfil parabólico, teniendo una velocidad máxima en el centro del canal de $1 \mathrm{~m} / \mathrm{s}$.

La Figura 2 muestra el coeficiente de presión $C_{p}$ resultante en la superficie del hidrofolio cuando $u_{\infty}=1$ $\mathrm{m} / \mathrm{s}$ y $\alpha=5^{\circ}$.

La influencia del angulo de ataque $\alpha$ también es in- vestigado. Cuando el angulo de ataque varía, $C_{p}$ se modifica considerablemente. Las Figures 3 y 4 muestran los resultados de la simulación para la distribución del coeficiente de presión alrededor del hidrofolio con diferentes ángulos de ataque.

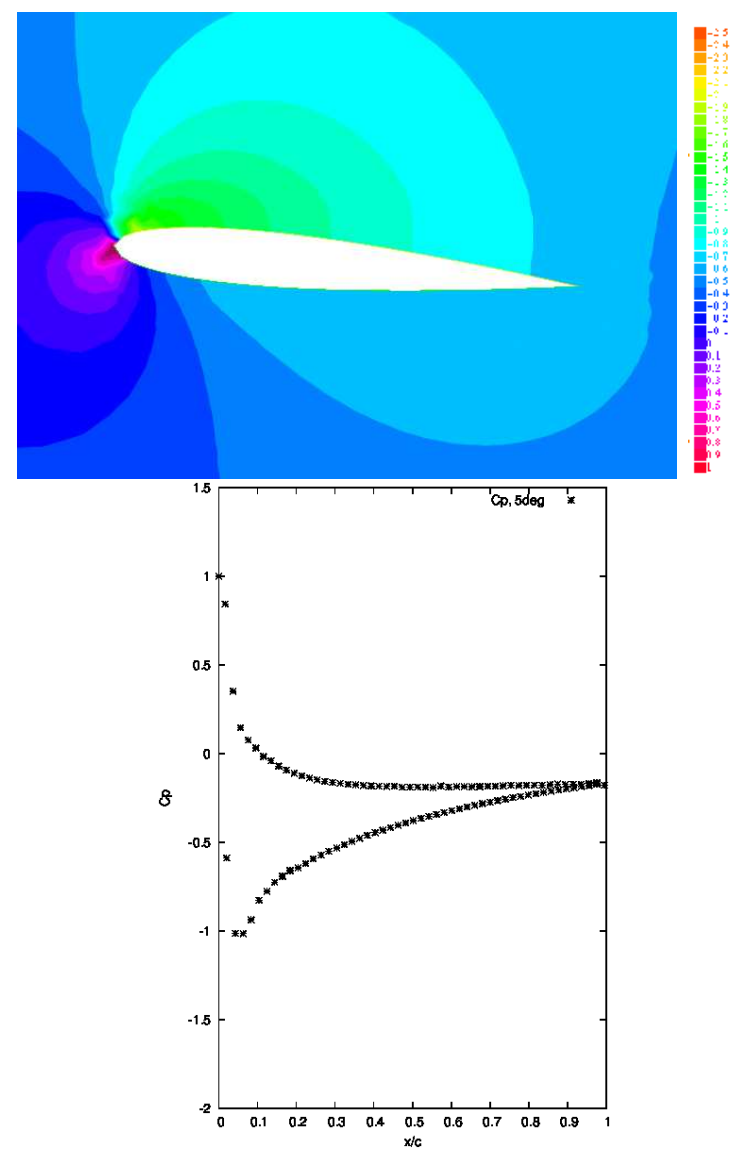

Figura 4: Campo de $C_{p}$. Arriba: para $\alpha=10^{\circ}, u_{\infty}=1$ $\mathrm{m} / \mathrm{s}$, prof $=2 \mathrm{c}$. Abajo: $C_{p}$ en la superficie del hidrofolio.

El aumento del angulo de ataque disminuye la presión en la parte superficial alcanzando valores cercanos de $C_{p}=-2$ para $\alpha=10^{\circ}$, mientras que para $\alpha=5^{\circ}$ el valor del coeficiente de presión es alrededor de $C_{p}=-1$.

El estudio de la distribución de la presión y la fracción de volumen de vapor en condiciones de cavitación se aborda seguidamente. Se considera un flujo mezclado con la densidad variando con la presencia del vapor de agua. Como datos, la densidad del vapor en condiciones de saturación es $\rho_{v}=0.02308 \mathrm{~kg} / \mathrm{m}^{3}$ y a $25^{\circ} \mathrm{C}$ $P_{v}=3169 \mathrm{~Pa}$. 


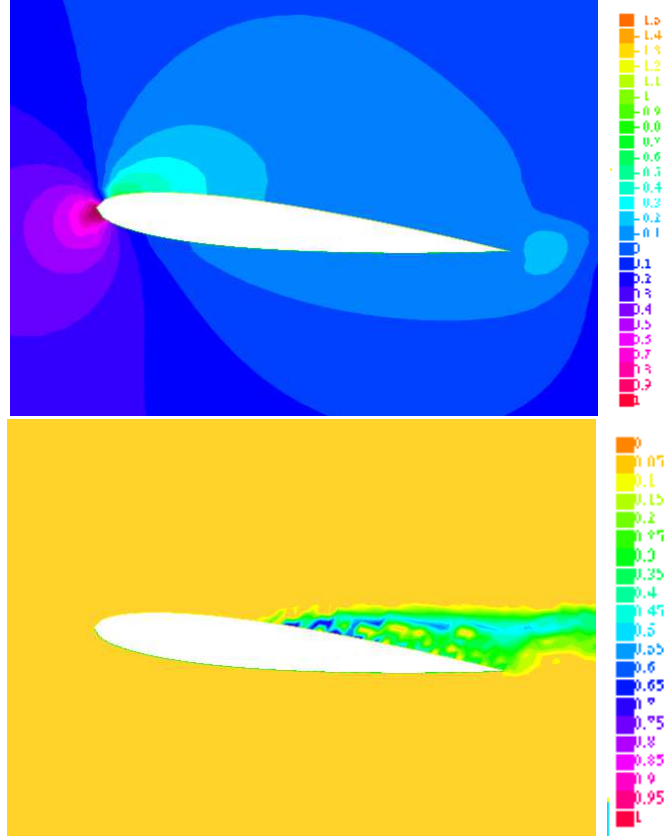

Figura 5: Distribución de $C_{p}$ (Arriba) y $\alpha_{v}$ (Abajo). Para $t=0.5 \mathrm{~s} \operatorname{con} \alpha=6^{\circ}, u_{\infty}=6 \mathrm{~m} / \mathrm{s}, \sigma=0.8$.

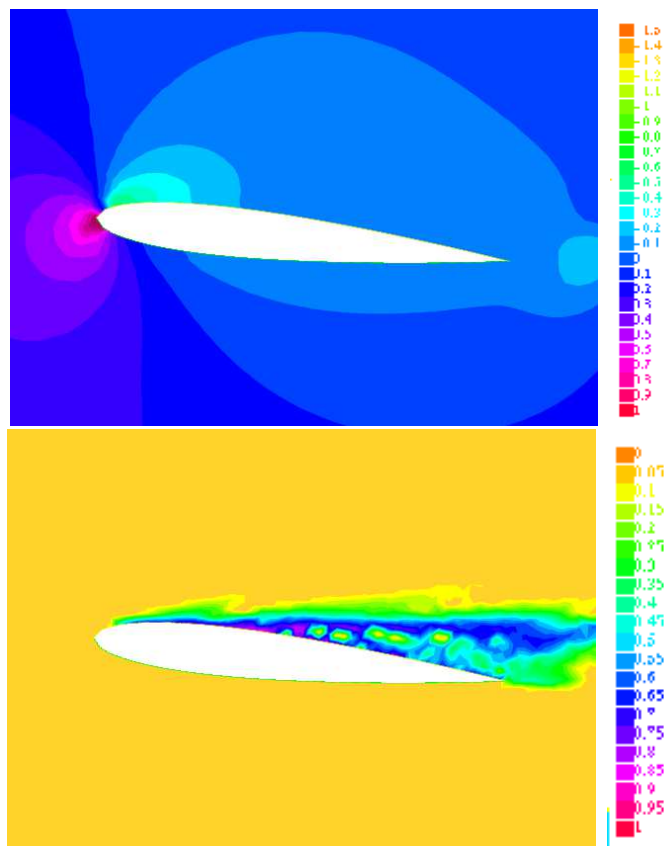

Figura 6: Distribución de $C_{p}$ (Arriba) y $\alpha_{v}$ (Abajo). Para $\mathrm{t}=0.5 \mathrm{~s}$ con $\alpha=6^{\circ}, u_{\infty}=6 \mathrm{~m} / \mathrm{s}, \sigma=0.4$.

Las condiciones de escurrimiento con cavitación se describen mediante el numero $\sigma$ definido como

$$
\sigma=\frac{p_{v}-p}{\frac{1}{2} \rho u_{\infty}^{2}}
$$

Los resultados transientes cuando se presenta el fenomeno de cavitación, se muestran en las Figuras 5 y 6 en el instante final $t=0.4 \mathrm{~s}$ para las distribuciones del coeficiente de presión $C_{p}$ y la fracción de volumen de vapor $\alpha_{v}$, cuando los números de cavitación $\sigma$ son iguales a 0.8 y 0.4 .

Las soluciones muestran la generación de vapor en la parte superficial del hidrofolio en donde la densidad disminuye con un consecuente comportamiento cavitante. Las cavidades de vapor de agua se inician en la parte superior del hidrofolio en un sector cerca del punto de ataque, a una distancia de un $10 \%$ de la cuerda del hidrofolio.

Durante la respuesta transitoria, las cavidades de vapor son transportadas en la dirección aguas abajo del hidrofolio. La mayor intensidad del volumen de vapor de agua está asociada a un bajo número de cavitación $\sigma$. Uno de los efectos en la respuesta dinámica, es que el coeficiente de presión negativo no es mayor por la formación de la cavidad de vapor de agua.

Las respuestas dinámicas obtenidas para los diversos casos considerados en esta sección, reproducen características observadas, presentando particularmente una fuerte variabilidad temporal durante la presencia del fenómeno de cavitación.

\section{Conclusiones}

Hemos propuesto un modelo para simular la hidrodinámica alrededor de hidrofolios y mostramos el estado actual de desarrollo que se ha incluido en el modelo.

La diferencia con otros modelos existentes está marcado por tres factores: 1) Es una formulación de elementos finitos; 2) El modelo de Prandtl Kolmogorov es usado para la turbulencia; 3) Un tipo de condición de frontera abierta débilmente reflectantte es incluído.

Los resultados de los experimentos numéricos han mostrado diversas condiciones de escurrimiento del fluido alrededor de un hidrofolio.

El modelo presentado permite la descripción de características dinámicas para condiciones de flujo a diferentes velocidades y a diferentes ángulos de aproximación de $5^{\circ}$ y $10^{\circ}$ verificandose el rol de los ángulos de inclinación en el coeficiente de presiones $C_{p}$ sobre el hidrofolio.

Experimentos transientes para condiciones de cavitación fueron analizados. Los resultados muestran una evolución transiente de la fracción de volumen de vapor $\alpha_{v}$ en la parte superior del hidrofolio influenciando directamente el coeficiente de presión negativo $C_{p}$. 


\section{Referencias}

[1] L. Prandtl y O. G. Tietjens; Hydro and Aeromechanics, Dover Publications, Nueva York (1957).

[2] H. Oertel; Essentials of Fluid Mechanics, Applied Mathematical Sciences, Springer Verlag, Berlin (2004).

[3] N. Mostafa, M. M. Karim y M. M. A. Sarker; $A$ study on numerical analysis of unsteady flow over two dimensional hydrofoils, 11-12 December 2010, BUET, Dhaka, Bangladesh (2010).

[4] M. Karim, N. Mostafa y M. Sarker; J. Naval Architecture \& Marine Engineering 7, 51 (2010).

[5] T. Kawamura y M. Sakoda; Fifth International Symposium on Cavitation, CAV2003, Osaka, Japan, November 1-4 (2003).
[6] T. Hughes; The Finite Element Method, Dover Publications, 682pp, New York (2000).

[7] J. Connor y C. Brebbia; Finite Element Techniques for Fluid Flow, Newnes-Butterworths, Londres (1980).

[8] C. Carbonel; Proceedings od 2nd International Congress of Mechanical Engineering, COBEM 2013, Ribeirão Preto, São Paulo, Brazil, November 3-7 (2013).

[9] A. Singhal, H. Li, M. M. Atahavale y Y. Jiang; J. Fluid Engineering 124, 617 (2002).

[10] M. Abbot; An Introduction to the Method of Characteristics, Thames-Hudson, New Tork (1966).

[11] O. Pironneau; Numerische Mathematik 38, 309 (1982).

[12] F. Hecht; J. Numer. Math. 20(3-4), 251 (2012). 